\title{
Association between platelet to lymphocyte ratio and plateletcrit with the presence of hyperemesis gravidarum: A case-control study
}

Abstract

Background and objective: Hyperemesis gravidarum can be defined as intractable nausea and vomiting, leading to electrolyte imbalance, ketonuria, nutrition deficiency, and weight loss during pregnancy. This study aimed to investigate the association of the platelet to lymphocyte ratio and plateletcrit with the presence of hyperemesis gravidarum.

Methods: A case-control study was conducted at Maternity Teaching Hospital in Erbil city, Kurdistan region of Iraq, between January 1st, 2017 and January 1st, 2018. A convenience sample of 120 pregnant women, of which 60 of them with a diagnosis of hyperemesis gravidarum admitted to the emergency department, were regarded as cases. The other 60 visited the outpatient department with mild discomfort, but not having hyperemesis gravidarum were regarded as controls. Age, gestational age, gravida, parity, height, and weight and laboratory parameters, including complete blood count from which we had platelet to lymphocyte ratio and plateletcrit \%, were recorded on a specially designed questionnaire. Chi-square test, Fisher's exact test, Student's t-test, Youden's index were used to determine the associations.

Results: The platelet to lymphocyte ratio and plateletcrit were higher in the hyperemesis gravidarum groups than controls $(P<0.001)$. The area under the curve for platelet to lymphocyte ratio and plateletcrit were 0.887 and 0.936 , respectively, with $P<0.001$. Platelet to lymphocyte ratio $>145.07$ and plateletcrit $>0.205 \%$ were significantly related to an increased risk of hyperemesis gravidarum.

Conclusion: The platelet to lymphocyte ratio and plateletcrit are higher in hyperemesis gravidarum cases and may have a predictive value of the development of hyperemesis gravidarum as further studies needed in the future to confirm.

Keywords: Hyperemesis gravidarum; Platelet to lymphocyte ratio; Plateletcrit; Pregnancy; Nausea and vomiting.

\section{Introduction}

Nausea and vomiting are very frequent complaints of pregnant women. Erroneously called "morning sickness" in $80 \%$ of these women, symptoms may continue throughout the day. Seventy- five percent of pregnant women express nausea and vomiting, lasting an average of 35 days. Half of them will report relief by 14 weeks and $90 \%$ by 22 weeks. ${ }^{1,2}$ Women with severe nausea and excessive vomiting during pregnancy have hyperemesis gravidarum (HG) which occurs in approximately $0.3-2 \%$ of pregnancies. ${ }^{3}$ Various HG definitions combine a number of symptoms that include protracted vomiting and nausea in pregnancy, accompanied by weight loss, disturbance of electrolyte balance, ketonuria, and dehydration or hospitalization; however, there are no unequivocal diagnostic criteria for HG as it is a diagnosis of exclusion. ${ }^{4}$ Risk factors reported to be associated with NVP and hyperemesis are low maternal age, first parity ${ }_{7}^{5}$ female offspring, ${ }^{6-8}$ multiple pregnancy, ${ }^{7}$ hyperthyroidism, molar pregnancy, gastrointestinal disorders,

${ }^{1}$ Department of Obstetrics and Gynecology, Maternity Teaching Hospital, Erbil, Iraq.

* Correspondence: naelafaraj1986@gmail.com 
gestational trophoblastic neoplasia, diabetes, and psychiatric disorders ${ }^{6}$ and history of $H G$ increases the risk of developing the disease to $15 \% .{ }^{9}$ Several possible mechanisms suggested to take part in the pathogenesis of $\mathrm{HG}$, one of them is inflammation and known to play a crucial role in HG etiopathogenesis. ${ }^{10}$ Severe chronic inflammation leads to increased proliferation in megakaryocytic series and relative thrombocytosis. In these cases, lymphocyte counts decrease as a result of severe apoptosis. Platelets are known to modulate inflammatory reactions beyond their role in blood coagulation and hemostasis. ${ }^{11-14}$ According to the researchers' best knowledge, this study is regarded as the first to be conducted in Maternity Teaching Hospital to determine the level of platelet to lymphocyte ratio (PLR) and plateletcrit (PCT) in cases of HG.

\section{Methods}

This case-control study was conducted at the Maternity Teaching Hospital in Erbil city, Kurdistan region of Iraq, between January 1st, 2017 and January 1st, 2018. A convenience sample of 120 pregnant women was enrolled. Sixty women with a diagnosis of HG who were admitted to the emergency department were regarded as cases. The other 60 visited the outpatient department with mild discomfort were regarded as controls. HG was diagnosed if the following symptoms were present: at least one positive ketonuria and severe vomiting more than twice a day, prior to the $24^{\text {th }}$ gestational week. ${ }^{4}$ The exclusion criteria included the existence of any disease related to inflammation such as hepatic, renal, or thyroid diseases, systemic or infectious diseases, gestational trophoblastic disease, gestational diabetes mellitus, pre-eclampsia, gastrointestinal disorders, metabolic disorders, collagen vascular diseases, smoking habits, alcohol consumption, urinary tract infection, and pregnancy with multiples. The PLR can be defined as the absolute platelet ratio to lymphocyte count obtained from the same blood sample. ${ }^{15,16}$ Similarly, PCT is a well-known parameter obtained from complete blood count (CBC). ${ }^{14}$ The sample size was calculated to be 58 for each group (58 for cases and 58 for control) by taking the level of significance of $5 \%$, power of study $80 \%$ and the standard deviation of PLR of 46 in the population in Iran, ${ }^{17}$ with a clinically important difference in mean PLR of $24 .{ }^{18}$ The sample was increased to 60 cases and 60 controls for convenience. Written informed consent was obtained from all individual participants included in this study after explaining to them the methods and the purpose of the study. A thorough history was taken from the patients to exclude those who were not going with the selected criteria for participation in the study. Then a general physical and systemic examination was performed. The data were recorded on a specially designed questionnaire arranged for this purpose to cover the age, gestational age, gravida, parity, height, and weight. The last menstrual period's start date was used to determine the gestational age, which was confirmed by first trimester sonography. Body mass index (BMI) was calculated as body weight $(\mathrm{kg})$ divided by the square of height $\left(\mathrm{m}^{2}\right)$. For the study group, laboratory investigations, including glucose, lipid profile, liver, and renal function tests, and electrolytes, were recorded. Blood samples for measurement of PLR and PCT and urine samples for determining the urine ketone levels were obtained from patients with $\mathrm{HG}$ at the time of hospitalization when the symptoms were most severe prior to intravenous hydration treatment. For the control group, all samples were collected during their prenatal visit for mild discomfort in the outpatient department. About $3.0 \mathrm{ml}$ of peripheral blood was drawn from each woman for complete blood cell count (CBC), and about $6.0 \mathrm{ml}$ for other laboratory tests. The procedure was done in the laboratory department of the hospital. Blood samples were collected 
in $\mathrm{K}_{2}$ EDTA $5.4 \mathrm{mg}$ containing tubes and processed in a Medonic M-Series hematology analyzer, an automated blood counter for $\mathrm{CBC}$ analysis. We recorded the following parameters from $\mathrm{CBC}$ records: platelet (PLT) count, 100 to $40010^{9} /$; plateletcrit (PCT), 0.08 to $0.44 \%$; lymphocyte count (LYM), 0.5 to $5.010^{9} /$; hemoglobin $(\mathrm{Hb}), 11.5$ to $16.5 \mathrm{~g} / \mathrm{dl}$; hematocrit (Hct), 35.0 to $55.0 \%$; white blood cells (WBC), 3.5 to $10.010^{9} /$; red blood cell distribution width (RDW), 11 to $16 \%$. The platelet count divided by absolute lymphocyte count (PLR) was calculated and compared between pregnant women with excessive vomiting and control groups, the same comparison done for plateletcrit (PCT). Ethical considerations: Approval was received for this study's proposal from the Ethics and Scientific Committee of Kurdistan Board for Medical Specialties (KBMS) and Maternity Teaching Hospital in Erbil city. All procedures involving human participants were performed in accordance with the ethical standards.

Statistical analysis: Data were analyzed using the statistical package for the social sciences (SPSS, version 22). The Chi-square test of association was used to compare proportions. Fisher's exact test was used when the expected count of more than $20 \%$ of the cells of the table was less than 5. Student's $t$ test of two independent samples was used to compare two means. Youden's index was calculated from the following equation: Youden's index = Sensitivity + specificity $1 .^{19}$ The highest value of Youden's index obtained from the study results was used as an indicator for the test cutoff point that gives us the highest sensitivity and the highest specificity values. A $P$ value of $\leq 0.05$ was considered statistically significant.

\section{Results}

The study sample was composed of sixty pregnant women with hyperemesis gravidarum (cases), and sixty pregnant women without such a condition (controls). Table 1 shows that the mean age \pm SD of the cases was $27.17 \pm 4.43$ years, and that of the controls was $27.12 \pm 5.67$ years $(P=0.957)$. The mean gestational age of the cases (13.58 weeks) was significantly $(P<0.001)$ higher than that of the controls (10.58 weeks). The table also shows that the means of parity and body mass index (BMI) were significantly higher among the controls than the cases.

Table 1: Means of the basic characteristics of the cases and controls.

\begin{tabular}{lccccc}
\hline & \multicolumn{2}{c}{ Cases } & \multicolumn{2}{c}{ Controls } & \\
& Mean & $( \pm$ SD) & Mean & $( \pm$ SD) & $P$ value \\
\hline Age (years) & 27.17 & $( \pm 4.43)$ & 27.12 & $( \pm 5.67)$ & 0.957 \\
Gestational age (weeks) & 13.58 & $( \pm 3.99)$ & 10.58 & $( \pm 2.61)$ & $<0.001$ \\
Parity (number) & 1.47 & $( \pm 1.14)$ & 2.00 & $( \pm 1.53)$ & 0.032 \\
BMl (kg/m2) & 20.70 & $( \pm 1.43)$ & 25.61 & $( \pm 1.91)$ & $<0.001$ \\
\hline
\end{tabular}


All the biochemical parameters of the cases and controls were within the normal range except for the chloride where $35 \%$ of the cases had low chloride level and none of the controls had such a condition $(P<0.001)$ as presented in Table 2.

Table 2: Biochemical parameters of the study groups.

\begin{tabular}{|c|c|c|c|c|c|c|c|}
\hline & \multicolumn{2}{|c|}{ Cases } & \multicolumn{2}{|c|}{ Controls } & \multicolumn{2}{|c|}{ Total } & \multirow[b]{2}{*}{$P$ value } \\
\hline & No. & $\begin{array}{c}(\%) \\
N=60\end{array}$ & No. & $\begin{array}{c}(\%) \\
\mathrm{N}=60\end{array}$ & No. & $\begin{array}{c}(\%) \\
\mathrm{N}=120\end{array}$ & \\
\hline Total cholesterol $(140-220 \mathrm{mg} / \mathrm{dl})$ & 60 & $(100.0)$ & 60 & $(100.0)$ & 120 & $(100.0)$ & NA \\
\hline $\mathrm{HDL}^{*}(40-60 \mathrm{mg} / \mathrm{dl})$ & 60 & $(100.0)$ & 60 & $(100.0)$ & 120 & $(100.0)$ & NA \\
\hline $\mathrm{LDL}^{*}(\leq 160 \mathrm{mg} / \mathrm{dl})$ & 60 & $(100.0)$ & 60 & $(100.0)$ & 120 & $(100.0)$ & NA \\
\hline Triglyceride $(35-200 \mathrm{mg} / \mathrm{dl})$ & 60 & $(100.0)$ & 60 & $(100.0)$ & 120 & $(100.0)$ & NA \\
\hline $\mathrm{GOT}^{*}(\leq 40 \mathrm{U} / \mathrm{L})$ & 60 & $(100.0)$ & 60 & $(100.0)$ & 120 & $(100.0)$ & NA \\
\hline $\mathrm{GPT}^{*}(\leq 40 \mathrm{U} / \mathrm{L})$ & 60 & $(100.0)$ & 60 & $(100.0)$ & 120 & $(100.0)$ & NA \\
\hline Alkaline phosphatase $(98-270 \mathrm{U} / \mathrm{L})$ & 60 & $(100.0)$ & 60 & $(100.0)$ & 120 & $(100.0)$ & NA \\
\hline Total bilirubin $(0.4-1.5 \mathrm{mg} / \mathrm{dl})$ & 60 & $(100.0)$ & 60 & $(100.0)$ & 120 & $(100.0)$ & NA \\
\hline Direct bilirubin $(\leq 0.4 \mathrm{mg} / \mathrm{dl})$ & 60 & $(100.0)$ & 60 & $(100.0)$ & 120 & $(100.0)$ & NA \\
\hline Blood urea (10-50 mg/dl) & 60 & $(100.0)$ & 60 & $(100.0)$ & 120 & $(100.0)$ & NA \\
\hline Serum creatinine $(0.6-1.4 \mathrm{mg} / \mathrm{dl})$ & 60 & $(100.0)$ & 60 & $(100.0)$ & 120 & $(100.0)$ & NA \\
\hline Glucose(70-115 mg/dl) & 60 & $(100.0)$ & 60 & $(100.0)$ & 120 & $(100.0)$ & NA \\
\hline Sodium (136-145 mEq/L) & 60 & $(100.0)$ & 60 & $(100.0)$ & 120 & $(100.0)$ & NA \\
\hline Potassium (3.5-5 mEq/L) & 60 & $(100.0)$ & 60 & $(100.0)$ & 120 & $(100.0)$ & NA \\
\hline \multicolumn{8}{|l|}{ Chloride (97-107 mEq/L) } \\
\hline$<97$ & 21 & $(35.0)$ & 0 & $(0.0)$ & 21 & $(17.5)$ & \\
\hline $97-107$ & 39 & $(65.0)$ & 60 & (100.0) & 99 & $(82.5)$ & $<0.001$ \\
\hline
\end{tabular}

*HDL, high density lipoprotein; LDL, low density lipoprotein; GOT, glutamic oxaloacetic transaminase; GPT, glutamic pyruvic transaminase. 
Table 3 shows that almost all of the sample had a normal lymphocyte count except for one case, but the difference between the groups was not significant $(P>0.999)$. The table shows that $15 \%$ of the cases had high platelet count, while none of the controls had such a high level $(P=0.003)$. All the women (cases and controls) had PCT\% between $0.08-0.44$, which is considered normal. All the women had hemoglobin $(\mathrm{Hb})$ levels lower than $11.5 \mathrm{~g} / \mathrm{dl}$. The other parameters mentioned in the table (Hct, WBC, and RDW) were within the normal range. It is evident in Figure 1 that the mean of the platelets / lymphocyte ratio among the cases (258.43) was significantly higher than the mean (110.97) of the control group $(P<0.001)$.

Table 3: Hematological parameters of the study groups.

\begin{tabular}{lccccccc}
\hline & \multicolumn{2}{c}{ Case } & \multicolumn{2}{c}{ Control } & \multicolumn{2}{c}{ Total } & \\
& No. & $(\%)$ & No. & $(\%)$ & No. & $(\%)$ & $P$ value \\
\hline Lymphocyte & & & & & & & \\
$(<0.5) 10^{9} / l$ & 1 & $(1.7)$ & 0 & $(0.0)$ & 1 & $(0.8)$ & \\
$(0.5-5) 10^{9} / l$ & 59 & $(98.3)$ & 60 & $(100.0)$ & 119 & $(92.2)$ & $>0.999^{*}$ \\
Platelets & & & & & & & \\
$(100-400) 10^{9} / l$ & 51 & $(85.0)$ & 60 & $(100.0)$ & 111 & $(92.5)$ & \\
$(>400) 10^{9} / l$ & 9 & $(15.0)$ & 0 & $(0.0)$ & 9 & $(7.5)$ & $0.003^{*}$ \\
PCT\% & & & & & & & \\
$(0.08-0.44) \%$ & 60 & $(100)$ & 60 & $(100.0)$ & 120 & $(100.0)$ & NA \\
Hb $(<11.5) g / d l$ & 60 & $(100.0)$ & 60 & $(100.0)$ & 120 & $(100.0)$ & NA \\
Hct $(35-55) \%$ & 60 & $(100.0)$ & 60 & $(100.0)$ & 120 & $(100.0)$ & NA \\
WBC $(3.5-10) 10^{9} / l$ & 60 & $(100.0)$ & 60 & $(100.0)$ & 120 & $(100.0)$ & NA \\
RDW $(11-16) \%$ & 60 & $(100.0)$ & 60 & $(100.0)$ & 120 & $(100.0)$ & NA \\
Total & 60 & $(100.0)$ & 60 & $(100.0)$ & 120 & $(100.0)$ & NA \\
\hline
\end{tabular}

*By Fisher's exact test

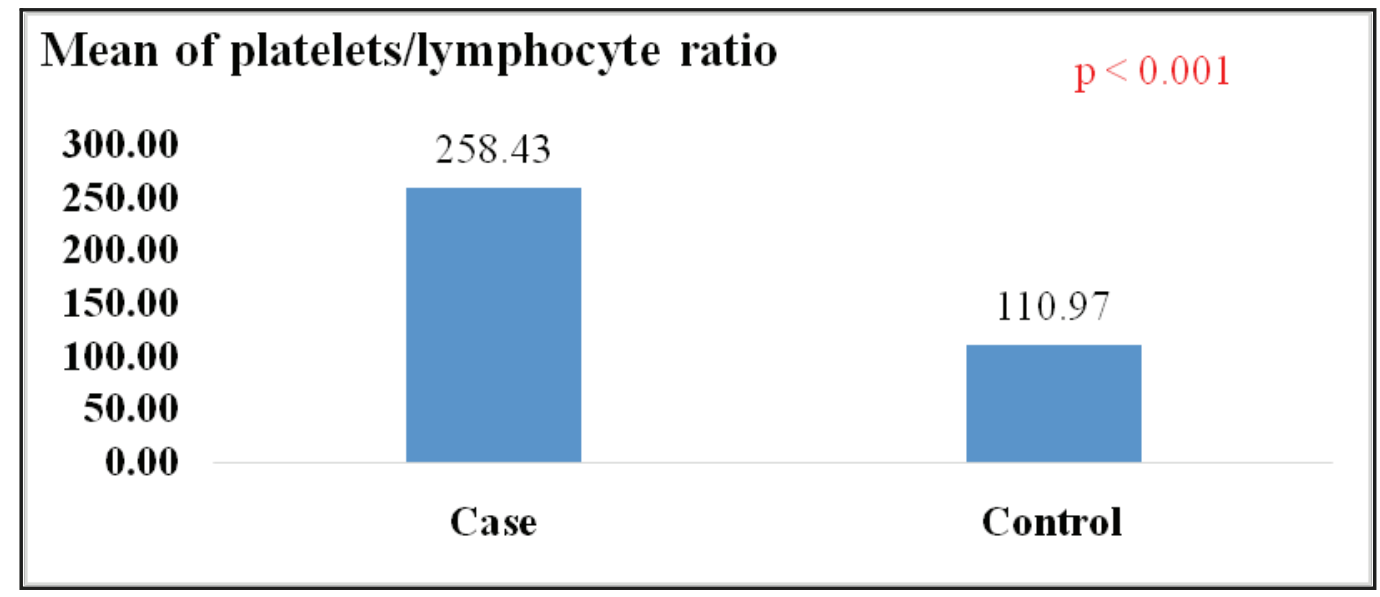

Figure 1: Means of platelets/lymphocyte ratio of the study groups. 
Figure 2 shows that the platelet/lymphocyte ratio and the $\mathrm{PCT} \%$ can significantly predict the HG $(P<0.001)$. The cutoff value for the platelet/lymphocyte ratio, as assessed according to the Youden's index was 145.07 , giving a sensitivity of $90 \%$ and a specificity of $78.3 \%$. The cutoff value for PCT\% was $0.205 \%$, giving a sensitivity of $85 \%$ and a specificity of $90 \%$.

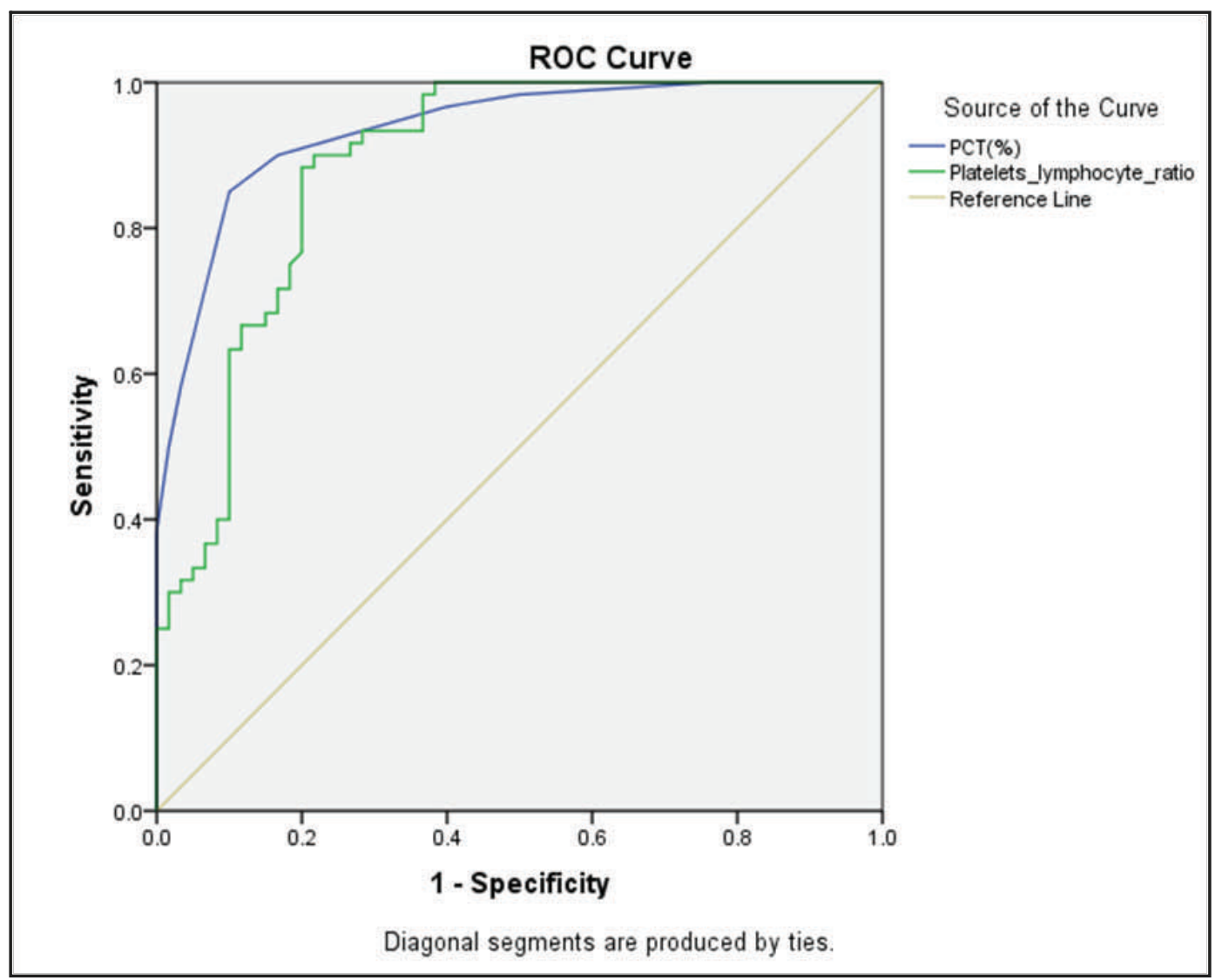

Figure 2: Receiver operator characteristic curve (ROC) testing the platelets lymphocyte ratio and $\mathrm{PCT} \%$ to detect hyperemesis gravidarum.

\section{Area under the curve}

\begin{tabular}{lcc}
\hline Test Result Variable (s) & $\begin{array}{c}\text { Platelets lymphocyte } \\
\text { ratio (PLR) }\end{array}$ & PCT (\%) \\
\hline Area under the curve & 0.887 & 0.936 \\
SE & 0.03 & 0.021 \\
$P$ value & $<0.001$ & $<0.001$ \\
Lower bound of 95\% Cl of the area & 0.828 & 0.894 \\
Upper bound of 95\% Cl of the area & 0.947 & 0.977 \\
Cut off value (according to Youden's index) & 145.07 & $0.205 \%$ \\
Sensitivity & $90 \%$ & $85 \%$ \\
Specificity & $78.3 \%$ & $90 \%$ \\
\hline
\end{tabular}




\section{Discussion}

The primary findings of our study demonstrated that an elevated PLR with a value of $>145.07$ and PCT $>0.205 \%$ were associated with the occurrence of HG. HG characterized by a number of symptoms that include protracted vomiting and nausea in pregnancy, accompanied by weight loss, disturbance of electrolyte balance, ketonuria, and dehydration or hospitalization, ${ }^{4}$ and may even progress in a highly fatal manner, leading to central pont in emyelinolysis and Wernicke's encephalopathy. ${ }^{20}$ Thus, early diagnosis of HG is crucial to prevent maternal and fetal mortality and morbidity. Inflammatory and immunologic mechanisms are thought to take part in the pathogenesis of $\mathrm{HG}^{21-23}$ The use of antihistamines ( $\mathrm{H} 1$ receptor antagonists) as a treatment modality led us to consider the effect of inflammatory processes in HG pathogenesis. ${ }^{24}$ Maternal histamine levels are increased under certain pathological circumstances, such as pre-eclampsia, spontaneous abortion, preterm labor and $\mathrm{HG}^{25}$ Starvation arises due to nausea and vomiting in patients with HG. The immune functions which are normally suppressed in the fasting state are active in pregnant women with HG. This suggests that immune mechanisms may be effective in the pathogenesis of the disease. ${ }^{10}$ Sometimes steroids are used in pregnant women with treatment-resistant nausea and vomiting and cause dramatic improvement. This suggests that the immune system and the inflammatory response may be active in the etiopathogenesis of $\mathrm{HG}^{26}$ The involvement of inflammatory processes in the development of HG has been studied from several aspects by different researchers. A positive correlation of interleukin-6 (IL-6) with $\beta$-human chorionic gonadotropin ( $\beta$-hcg) levels and increased levels of IL-6 in HG has been demonstrated. ${ }^{26}$ Tumor necrosis factor- $\alpha$, another inflammatory cytokine, is increased in $\mathrm{HG}^{27}$ Moreover, the ratio of Thelper (Th)1 cells to Th2 cells in patients with HG has been investigated, and an increase in Th2 cells was observed in the study group. ${ }^{23}$ Other studies have demonstrated significantly higher immunoglobulin(lg) G, IgM, C3, C4 levels and increased natural killercells, together with extra-thymic $T$ cells, in cases of $\mathrm{HG}^{28,29}$ In a study of serum soluble urokinase-type plasminogen activator receptor (suPAR) level was found to be significantly higher in pregnant women diagnosed with $H G$ in the first trimester compared to the control group. ${ }^{30}$ Adenosine deaminase (ADA) activity has been shown to be significantly increased in patients with $\mathrm{HG}^{31}$ Oxidative stress, also known to be related to inflammatory processes, is emphasized in HG. In their study, Verit et al. showed decreased levels of paranoxanase- 1 in cases of HG, which is another determinant of inflammatory processes involved in $\mathrm{HG}^{22}$ The use of vaspin, a novel pro-inflammatory adipocytokine, has been studied and the results revealed that vaspin was increased in patients with HG, which confirms the contribution of inflammatory processes in HG development. ${ }^{21} \mathrm{~A}$ study investigating the relationship between high sensitive C-reactive protein levels, neutrophil count, lymphocyte count, and NLR values reported that the NLR was significantly higher in the HG than in the control group and the NLR was directly correlated with HG severity. ${ }^{32}$ The PLR is implicated in several malignant, inflammatory and thrombotic processes. ${ }^{11,33}$ PCT is also known to be associated with several inflammatory conditions, such as Crohn's disease, coronary artery diseases, and some malignancies. ${ }^{11-13}$ There is no data in the literature regarding the role of the PLR and PCT in HG. Our study demonstrated that an elevated PLR with a value of 145.07 and PCT with a value of $0.205 \%$ were associated with the occurrence of HG. Both the PLR and PCT were found to be important independent discriminators for HG. However, an increased PCT value may result from dehydration, which is a clinical situation commonly occurring in 
HG cases. A study done in Bursa city in Turkey investigated the NLR, PLR values, and PCT and compared them in three groups divided into mild, moderate, and severe according to the severity of HG. It reported that these are inflammatory markers that are increased in patients with $\mathrm{HG}$ and have a predictive value of the development of $\mathrm{HG}$ in which NLR >3.9, PLR $>121.2$, and PCT $>0.20$ are associated with an increased risk of HG. Moreover, PCT increased with HG severity, $\mathrm{PCT}>0.20$ is related to $\mathrm{HG}$ severity. Increasing PCT values were not correlated with increasing urine density in any of the HG groups. ${ }^{18}$ Urinary ketone is a commonly used marker for diagnosis and decision-making regarding the severity of HG. We do not claim that the PLR and PCT are alternatives to urinary ketone in the diagnosis and management of HG. Our aim is to shed some light on the pathogenesis of $H G$ and emphasize its relationship with inflammatory processes by investigating its association with the PLR and PCT. Based on these results, the difference in PLR and PCT levels in the HG group can be considered to be associated with the etiopathogenesis of HG. There are some limitations to the current study. This study had a case-control design, and we only have the $C B C$ results from patients at the time of hospitalization. Therefore, PLR and PCT data after symptoms improved were not available. Moreover, we did not investigate the association between the urinary ketone and the PLR and PCT. Larger sample sized and prospective studies are required to compare the predictive role of each marker.

\section{Conclusion}

The PLR and PCT are inflammatory markers that are increased in patients with HG and have a predictive value of the development of HG. Because the association of these markers with $\mathrm{HG}$ has not been definitively investigated, data of these markers prior to $\mathrm{HG}$ development and according to the severity of $H G$ is not recorded. We cannot yet make a conclusive statement about their predictivity in clinical practice. As further data of the relationship between $\mathrm{HG}$ and inflammation is collected, the PLR and PCT may be proven as markers of HG in the future.

\section{Competing interests}

The authors declare no competing interests.

\section{References}

1. Cunningham FG, Leveno KJ, Bloom SL, Hauth JC, Gilstrap III L, Wenstrom KD. Prenatal care. In: Cunningham FG, Leveno KJ, Bloom SL, Hauth JC, Gilstrap III L, Wenstrom KD (eds) William's obstetrics. New York, NY:McGraw -Hill Medical Publishing Division; 2005. P. 20129.

2. Lacroix R, Eason E, Melzack R. Nausea and vomiting during pregnancy: a prospective study of its frequency, intensity, and patterns of change. Am J Obstet Gynecol 2000; 182:931-7.

3. Bailit JL. Hyperemesis gravidarium: epidemiologic findings from a large cohort. Am J Obstet Gynecol 2005; 193:811-4.

4. Niebyl JR. Clinical practice. Nausea and vomiting in pregnancy. N Engl J Med 2010; 363:1544-50.

5. Källen B, Lundberg G, Aberg A. Relationship between vitamin use, smoking, and nausea and vomiting of pregnancy. Acta Obstet Gynecol Scand 2003; 82:916-20.

6. Fell DB, Dodds L, Joseph KS, Allen VM, Butler B. Risk factors for hyperemesis gravidarum requiring hospital admission during pregnancy. Obstet Gynecol 2006; 107:277-84.

7. Basso O, Olsen J. Sex ratio and twinning in women with hyperemesis or pre-eclampsia. Epidemiology 2001; 12:747-9.

8. Schiff MA, Reed SD, Daling JR. The sex ratio of pregnancies complicated by hospitalization for hyperemesis gravidarum. BJOG 2004; 111:2730.

9. Trogstad LI, Stoltenberg C, Magnus P, Skjaerven $\mathrm{R}$, Irgens LM. Recurrence risk in hyperemesis gravidarum. $\mathrm{Br} \mathrm{J}$ Obstet Gynaecol 2005; 112:1641-5.

10. Verberg MF, Gillott DJ, Al-Fardan N, Grudzinskas JG. Hyperemesis gravidarum, a literature review. Hum Reprod Update 2005; 11:527-39.

11. Akpinar I, Sayin MR, Gursoy YC, Karabag T, Kucuk E, Buyukuysal MC, et al. Plateletcrit: A platelet marker associated with saphenous vein graft disease. Herz 2014; 39:142-8.

12. Nording HM, Seizer $P$, Langer HF. Platelets in inflammation and atherogenesis. Front Immunol 2015; 6:98. 
13. Kim CH, Kim SJ, Lee MJ, Kwon YE, Kim YL, Park KS, et al. An increase in mean platelet volume from baseline is associated with mortality in patients with severe sepsis or septic shock. PLoS One 2015; 10:e0119437.

14. Tang J, Gao X, Zhi M, Zhang M, Chen HW, Yang QF, et al. Plateletcrit: A sensitive biomarker for evaluating disease activity in Crohn's disease with low hs-CRP. J Dig Dis 2015; 16:118-24.

15. Akbas EM, Demirtas L, Ozcicek A, Timuroglu A, Bakirci EM, Hamur $\mathrm{H}$, et al. Association of epicardial adipose tissue, neutrophil-tolymphocyte ratio and platelet-to-lymphocyte ratio with diabetic nephropathy. Int J Clin Exp Med 2014; 7:1794-801.

16. Balta $S$, Ozturk $C$. The platelet-lymphocyte ratio: A simple, inexpensive and rapid prognostic marker for cardiovascular events. Platelets 2014; $30: 1-2$

17. Terzi S, Dursun E, Özgür A, Yiğit E, Özergin-Coşkun Z, Çelebi-Erdivanl O, et al. Status of Neutrophils, Lymphocytes and Platelets in Patients with Recurrent Aphthous Stomatitis: A Retrospective Study. Iran J Otorhinolaryngol 2016; 28(89):421-4.

18. Tayfur C, Burcu DC, Gulten O, Betul D, Tugberk $\mathrm{G}$, Onur O, et al. Association between platelet to lymphocyte ratio, plateletcrit and the presence and severity of hyperemesis gravidarum. J Obstet Gynaecol Res 2017; 43(3):498-504.

19. Bewick V, Cheek L, Ball J. Receiver operating characteristic curve. Critical care 2004; 8(6):50812.

20. Netravathi M, Sinha S, Taly AB, Bindu PS, Bharath RD. Hyperemesis-gravidaruminduced Wernicke's encephalopathy: Serial clinical, electrophysiological and MR imaging observations. J Neurol Sci 2009; 284:214-6.

21. Engin-Ustun $Y$, Tonguç $E$, Var $T$, Deveer $R$, Yilmaz N, Danisman $N$, et al. Vaspin and C-reactive protein levels in hyperemesis gravidarum. Eur Rev Med Pharmacol Sci 2013; 17:138-40.

22. Verit FF, Erel O, Celik H. Paraoxonase-1 activity in patients with hyperemesis gravidarum. Redox Rep 2008; 13:134-8.

23. Yoneyama Y, Suzuki S, Sawa R, Yoneyama K, Doi D, Otsubo Y, et al. The T-helper 1/T-helper 2 balance in peripheral blood of women with hyperemesis gravidarum. Am J Obstet Gynecol 2002; 187:1631-5.

24. Jarvis S, Nelson-Piercy C. Management of nausea and vomiting in pregnancy. BMJ 2011; 342:d3606.

25. Brew O, Sullivan MH. The links between maternal histamine levels and complications of humanpregnancy. J Reprod Immunol 2006; 72:94 $-107$.

26. Kuscu NK, Yildirim Y, Koyuncu F. Interleukin-6 levels in hyperemesis gravidarum. Arch Gynecol Obstet 2003; 269:13-5.
27. Kaplan PB, Gucer F, Sayin NC, Yüksel M, YüceMA, Yardim T. Maternal serum cytokine levels in women with hyperemesis gravidarum in the first trimester of pregnancy. Fertil Steril 2003; 79:498-502.

28. Leylek OA, Toyaksi M, Erselcan T, Dokmetas S. Immunologic and biochemical factors in hyperemesis gravidarum with or without hyperthyroxinemia. Gynecol Obstet Invest 1999; 47:229-34.

29. Minagawa M, Narita J, Tada T, Maruyama S, Shimizu $T$, Bannai $M$, et al. Mechanisms underlying immunologic states during pregnancy: Possible association of the sympathetic nervous system. Cell Immunol 1999; 196:1-13.

30. Desdicioglu R, Yildirim M, Kocaoglu G, Cendek BD, Avcioglu G, Tas EE, et al. Soluble urokinase-type plasminogen activator receptor (suPAR) and interleukin-6 levels in hyperemesis gravidarum $\mathrm{J}$ Chin Med Assoc 2018; 81(9):825-9.

31. Yoneyama $\mathrm{Y}$, Sawa $\mathrm{R}$, Suzuki S, Otsubo $\mathrm{Y}$, Araki T. Serum adenosine deaminase activity in women with hyperemesis gravidarum. Clin Chim Acta 2002; 324:141-5.

32. Keskin KR, Guler A, BenkSilfeler D, Ozcil MD, Karateke A, Hakverdi AU. Relation of inflammatory markers with both presence and severity of hyperemesis gravidarum. Ginekol Pol 2014; 85:589-93.

33. Wang D, Yang JX, Cao DY, Wan XR, Feng FZ, Huang HF, et al. Preoperative neutrophil-lymphocyte and platelet-lymphocyte ratios as independent predictors of cervical stromal involvement in surgically treated endometrioid adenocarcinoma. Onco Targets Ther 2013; 6:211-6. 